\title{
Final Report for A Mathematical Analysis of Atomistic-to-Continuum (AtC) Multiscale Coupling Methods
}

\author{
DE-FG02-05ER25698 \\ Max Gunzburger \\ Department of Scientific Computing \\ Florida State University \\ gunzburg@f su . edu
}

We have worked on several projects aimed at improving the efficiency and understanding of multiscale methods, especially those applicable to problems involving atomistic-tocontinuum coupling. Here, we briefly describe these activities.

\section{Blending methods for AtC coupling}

Continuum material models break down in regions where singular behavior, e.g., dislocations, crack tips, nano-indentations, etc., occur. Although sophisticated, nonlinear continuum models have been advanced to account for some of these microscopic phenomena, in truth, they are best modeled using atomistic models, e.g., molecular statics and dynamics. Away from places of singular behavior, it would be wasteful to use atomistic models since continuum models are known to be accurate. Thus, we examine situations for which a material is divided into two subdomains; in one, an atomistic model is used and in the other, a continuum model is used. The crucial algorithmic and mathematic question is then how should these models be coupled?

We have extensively studied the modeling, implementation, and analysis aspects of blending methods for effecting atomistic to continuum (AtC) coupling. In this approach, a third region, a buffer, is introduced between the atomistic and continuum domains; in the buffer region, both models are assumed valid but instead of simply applying both models, the two models are blended in such a way that near the atomistic region, the atomistic model is more "dominant" while the reverse is true near the continuum region. This approach provides for a smooth transition between the two models and greatly reduces deleterious effects such as ghost forces that are problematic with other coupling approaches.

Our work first focused on developing refinements of the blending model [1,2]. We have shown, for example, that some other blending models introduced in the literature violate Newton's third law of motion but that our models do not. We have also subjected $[2,3]$ various models to simple analytic patch tests; passing such tests are crucial to demonstrating that models accurately reflect the physics and also to the accuracy of discretization methods. We have also developed and analyzed [3] an abstract framework in which many, if not most, 
existing as well as some new forced-based blending models for AtC coupling are special cases. The abstract framework allows for a unified treatment of many methods through rigorous mathematical analyzes and also allows for a comparative study of the advantages and disadvantages of the various cases. We find that the new blending methods we have proposed and studied are, in many respects, superior to others that have been previously advanced.

In the past, a separation has been made between blending methods into those that are force-based and those that are energy-based. In further work, we reconciled these two approaches. As a result, most blending methods can be put into a single framework, showing that the separation is artificial, and enabling further insightful analyses. This work is reported on in [4].

Several issues connected with bridging methods for atomistic-to-continuum (AtC) were examined in [5]. Different coupling approaches using various energy blending models are studied as well as the influence that model parameters, blending functions, and grids have on simulation results. We used the Lagrange multiplier method for enforcing constraints on the atomistic and continuum displacements in the bridge region. We also show that continuum models are not appropriate for dealing with problems with singular loads, whereas AtC bridging methods yield correct results, thus justifying the need for a multiscale method. We investigate models that involve multiple-neighbor interactions in the atomistic region, particularly focusing on a comparison of several approaches for dealing with Dirichlet boundary conditions.

\section{Efficient quasi-continuum methods for problems with long-range interac- tions}

In certain situations, quasi-continuum methods are a means for reducing the cost of atomistic, e.g., molecular statics, calculation. As such, quasi-continuum methods are, strictly speaking, not AtC coupling methods in that there is no bona fide continuum model invoked. Rather, continuum notions, most notably finite element interpolation, are used to reduce the number of unknowns in a molecular statics computation. In the quasi-continuum approach, a grid is superimposed on the atomistic region; a particle is located at each node of the grid but there are (many) other particles located within each grid cell. The latter set of particles are slaved through (usually piecewise linear) interpolation to the master particles, i.e., those located at the nodes of the grid. In this way, the number of degrees of freedom are greatly reduced.

However, to make quasi-continuum methods truly efficient, one also has to do something about force determination since such calculations still require one to account for all the particles, not just the master particles. Thus, the computational costs remain dependent on the total number of particles and not just on the number of master particles. Several clustering and other methods have been advanced for this purpose, i.e., for rendering the total work independent of the total number of particles.

The force calculation require a double summation, with the outer one being over all the particles and the inner one being over the particles that interact with each particle in the outer sum. For short range interactions between particles, i.e., particles exert a force only on nearby particles, the inner sum is short whereas the outer sum remains long and causes costs to depend on the total number of particles and not on the much smaller 
number of representative particles. We have developed [6] a novel quadrature-rule type approximation for the long outer sum; in this approach, the outer sum is approximated by a much shorter weighted sum over a few of the slave points within each triangle in much the same way integrals are approximated by sums over few quadrature points. Simple analyses and computational experiments were used to illustrate that the new method has, for the same accuracy, lower complexity compared to not only the quasi-continuum method, but also to cluster-type approximations of that method. Implementation issues connected to the new method has also been provided.

Existing quasi-continuum methods (that include efficient means for force calculations) are only efficient and accurate for problems for which the forces have short-range effects, e.g., particles exert a force only on nearby particles. In many situations, e.g., when Coulomb forces are present, such an assumption is not valid. In the case of long-range interactions, both the inner and outer sums in the force determination are long. The reason why many existing methods fail to make costs depend on the number of representative particles and not on the total number of particles is that they only address the outer summation. We have developed [7] a novel quadrature-rule type approximation that addresses both the inner and outer sums. As a result, even for the long-range interaction cases, the complexity of this method depends on the number of representative particles but not on the total number of particles. Simple analyses and numerical experiments are used illustrate the accuracy and performance of the method.

\section{Junior researchers supported by grant}

Postdoctoral associate: Yanzhi Zhang current position: Assistant Professor, Missouri Institute of Science and Technology

Ph.D. student: Pablo Seleson current position: ICES Postdoc, Univesity or Texas at Austin

\section{References}

[1] J. Fish, M. Nuggehally, M. Shephard, C. Picu, S. Badia, M. Parks, and M. GunzBurger, Concurrent AtC coupling based on a blend of the continuum stress and the atomistic force, Computer Methods in Applied Mechanics and Engineering, 196 2007, 4548-4560.

[2] S. Badia, P. Bochev, J. Fish, M. Gunzburger, R. Lehoucq, M. Nuggehally, AND M. PARKS, A force-based blending model for atomistic-to-continuum coupling, International Journal for Multiscale Computational Engineering, 5 2007, 387-406.

[3] S. Badia, M. Parks, P. Bochev, M. Gunzburger, and R. Lehoucq, On atomistic-to-continuum (AtC) coupling by blending, Multiscale Modeling and Simulation, 7 2008, 381-406.

[4] S. Badia, P. Bochev, M. Gunzburger, R. Lehoucq, and M. Parks, Blended energy methods for atomistic-to-continuum coupling, in Multiscale Methods: Bridging the Scales in Science and Engineering, Oxford, Oxford, 2010, 165-189. 
[5] P. Seleson and M. Gunzburger, Bridging methods for atomistic-to-continuum coupling and their implementation, Communications in Computational Physics, 7 2010, 831-876.

[6] M. Gunzburger And Y. Zhang, A quadrature-rule type approximation to the quasicontinuum methods, Multiscale Modeling and Simulation, 8 2010, 571-590.

[7] Y. Zhang And M. GunzBurger, Quadrature-rule type approximations to the quasicontinuum method for long-range interatomic interactions, Computer Methods in Applied Mechanics and Engineering, 199 2010, 648-659. 